\title{
Systemic inflammation in both open-angle and angle-closure glaucoma: role of platelet-to-lymphocyte ratio
}

\author{
Karahan $\mathrm{M}^{1}$, Kilic $\mathrm{D}^{2}$, Guven $\mathrm{S}^{2}$ \\ Dicle University Faculty of Medicine, Department of Ophthalmology, Diyarbakir, Turkey. \\ dnz_kilic@hotmail.com
}

\section{ABSTRACT}

AIM: To analyse the effect of systemic inflammatory status in patients with primary open-angle glaucoma (POAG) and primary angle-closure glaucoma (PACG) by calculating platelet-to-lymphocyte ratio (PLR) and neutrophil-to-lymphocyte ratio (NLR).

METHODS: This retrospective case-control study included 200 patients with POAG, 22 patients with PACG and 100 healthy subjects. The participants' white-blood-cell, lymphocyte, neutrophil, and platelet counts were recorded from previous blood assays. NLR and PLR were calculated manually. Results were compared among the groups.

RESULTS: Both the POAG and PACG groups had higher platelet counts and PLR values than the control group ( $p=0.001$ and $p=0.001$; respectively). The difference in NLR between POAG, PACG and control groups was not statistically significant $(p=0.076)$. The POAG group had higher NLR values than the control $(p=0.035)$. CONCLUSION: Both the POAG and the PACG groups exhibited higher platelet and PLR levels than the control. These results indicate a potential role of systemic inflammation in the pathogenesis of POAG and PACG (Tab. 4, Fig. 1, Ref. 35). Text in PDF www.elis.sk

KEY WORDS: neutrophil-to-lymphocyte ratio; platelet-to-lymphocyte-ratio; primary open-angle glaucoma; primary angle-closure glaucoma; systemic inflammation.

\section{Introduction}

Glaucoma is thought to be amongst the major causes of irreversible blindness worldwide and it is composed of groups of progressive optic neuropathies (1-3). While high intraocular pressure (IOP) is a well-known treatable risk factor, discussion remains regarding the exact mechanism of both primary open-angle glaucoma (POAG) and primary angle-closure glaucoma (PACG) (3-5). Notably, vascular dysfunction, autoimmune processes, impaired systemic oxidation/anti-oxidation balance and inflammatory pathways are blamed as underlying molecular mechanisms (6-11). Therefore, there could be a role of systemic inflammation in the etiopathogenesis of glaucoma.

One of the most inexpensive and simplest ways to evaluate systemic inflammation is the calculation of platelet-to-lymphocyte ratio (PLR) and neutrophil-to-lymphocyte ratio (NLR) (12). These parameters are obtained from a complete blood count $(\mathrm{CBC})$ and have widely been used as predictors in the prognosis of several systemic diseases (13-18). It has also been noted that favourable outcomes could be achieved by using both PLR and NLR in ocu-

${ }^{1}$ Dicle University Faculty of Medicine, Department of Ophthalmology, Diyarbakir, Turkey, and ${ }^{2}$ Health Science University, Kayseri City Hospital, Department of Ophthalmology, Kayseri, Turkey

Address for correspondence: D. Kilic, MD, FEBO, Kayseri Training and Research Hospital, Department of Ophthalmology, Kayseri, Turkey. Phone: +90.505.9203928, Fax: +90.352.3157700 lar pathologies (19-22). However, there are few existing studies in the literature that have compared both PLR and NLR in glaucoma patients (23-25).

In this present study, our purpose was to evaluate whether there is an effect of systemic inflammation by comparing the values of PLR and NLR between the patients with any types of glaucoma (POAG and/or PACG) and healthy subjects.

\section{Patients and methods}

\section{Study population}

This was a retrospective case-control study performed at the Dicle University Department of Ophthalmology in Diyarbakir, Turkey. Digital medical records between January 2010 and February 2019 were used to obtain the research data. The Dicle University Review Board approved the methodology. The study adhered to the Declaration of Helsinki for clinical research.

The patients were all followed up in the Department of Glaucoma. A total of 306 patients' files were searched. We excluded 54 patients for the lack of detailed ophthalmological examination data, while 30 additional patients were excluded for the absence of $\mathrm{CBC}$ results. Finally, 200 patients with POAG, 22 patients with PACG and 100 gender-matched subjects who were otherwise healthy were enrolled in the study as controls. Controls were individuals who were arranged for routine cataract surgery and their $\mathrm{CBC}$ was obtained during preoperative laboratory assessments (Fig. 1). 


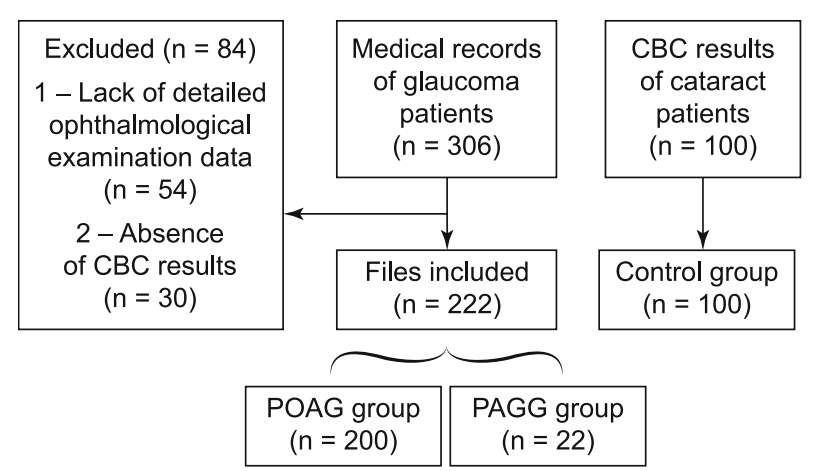

Fig. 1. Flow of inclusion criteria.

A detailed ophthalmological examination was performed, including best corrected visual acuity (BCVA) measurements with Snellen charts, anterior and posterior segments evaluation by slitlamp biomicroscopy and IOP measurements using a Goldmann applanation tonometer (Haag-Streit Inc., Köniz, Switzerland). In our glaucoma department, if we diagnose a patient for any type of glaucoma, we routinely perform a complete blood analysis, including $\mathrm{CBC}$, biochemistry and hepatitis markers from all the patients on the same day.

\section{Clinical evaluation of glaucoma}

POAG was diagnosed if the anterior chamber angle was open on the gonioscopy with glaucoma-related optic neuropathy (GON), IOP of $>21 \mathrm{mmHg}$ and glaucoma-related visual field defect (GVFD).

PACG was defined as follows: the angle is occludable and/or the peripheral iris is capable of obstructing the angle on gonioscopy, GON, and GVFD and an IOP of $>21 \mathrm{~mm} \mathrm{Hg} \mathrm{(26).}$

GON was defined as an increase in optic cup excavation, neuro-retinal rim thinning, notching, haemorrhages on optic disc head, or cup/disc ratio (CDR) asymmetry among the eyes $(27,28)$.

In the Department of Glaucoma, a Humphrey Field Analyser was used to perform visual field tests (VFTs) (Carl Zeiss Meditec Inc., Dublin, CA). VFTs were completed using the Swedish Interactive Threshold Algorithm (SITA) Standard strategy, programme 30-2. Typical GVFDs were identified if at least in two reliably performed consecutive tests (SITA, 30-2 VFTs) in an individual have an abnormal glaucoma hemifield test or pattern standard deviation.

Tab. 1. Comparison of demographic and laboratory parameters between three groups

\begin{tabular}{lcccc}
\hline Variables & POAG $(\mathrm{n}=200)$ & PACG $(\mathrm{n}=22)$ & Control $(\mathrm{n}=100)$ & $\mathrm{p}$ \\
\hline Age $(\mathrm{y})$ & $70(40-85)$ & $57(42-89)$ & $67(41-86)$ & $0.001^{*}$ \\
Sex $(\mathrm{male} / \mathrm{female})$ & $92 / 108$ & $7 / 15$ & $49 / 51$ & 0.342 \\
WBC $\left(10^{3} / \mathrm{mL}\right)$ & $6.40(4-15)$ & $7.06(4.77-8.25)$ & $6.66(4.57-9.81)$ & 0.237 \\
Neutrophil $\left(10^{3} / \mathrm{mL}\right)$ & $3.81(2.03-11.00)$ & $4.25(2.51-6)$ & $4.03(2.09-7.60)$ & 0.249 \\
Lymphocyte $\left(10^{3} / \mathrm{mL}\right)$ & $1.80(0.61-3.88)$ & $1.87(1.04-2.96)$ & $1.96(0.83-2.13)$ & 0.089 \\
Platelet $\left(10^{3} / \mathrm{mL}\right)$ & $261(121-520)$ & $256.50(204-329)$ & $234.50(106-359)$ & $0.001^{*}$ \\
NLR & $2.19(0.79-13.23)$ & $2.27(1.39-3.88)$ & $2.08(0.20-6.73)$ & 0.076 \\
PLR & $147.77(61.63-412.9)$ & $139.63(82-269.2)$ & $109.95(12.30-304.8)$ & $0.001^{*}$ \\
\hline
\end{tabular}

Data are expressed as the median ( $\min -\max )$ or number of cases as appropriate. p-values were calculated using Kruskal-Wallis, Mann-Whitney U test and $\chi 2$ as appropriate. $\mathrm{n}=$ the number of patients; POAG, primary open angle glaucoma; PACG, primary angle closure glaucoma; NLR, neutrophil-to-lymphocyte ratio; PLR, plateletto-lymphocyte ratio; WBC, white blood cells. $* \mathrm{p}<0.05$
Abnormal VFTs met a minimum one of the following three criteria: $\geq 3$ nearby points with $p<0.05$ or greater loss; $\geq 2$ adherent points with $\mathrm{p}<0.01$ or greater loss; or a $10 \mathrm{~dB}$ dissimilarity across the nasal horizontal midline in $\geq 2$ adjacent locations compared to perimeter-defined gender-matched normal values (26).

\section{Blood sampling}

The antecubital vein was used to obtain the blood samples, and the measurements were done at the Biochemistry Department on the day of the diagnosis was made. The values of red blood cells (RBC), white blood cells (WBC), neutrophils, lymphocytes and platelets were measured in CBC tests (BC-6800, Mindray, Shenzhen, China). PLR and NLR were calculated manually.

\section{Statistical analysis}

Statistical Package for the Social Sciences (SPSS) 20.0 version was used for statistical analysis on a Windows-based PC. In our study, 'median (min-max)' and 'percentage (\%)' were used among the descriptive statistics. The Kolmogorov-Smirnov test was used to evaluate the distribution of variables. Quantitative values were compared between three groups with the KruskalWallis test. Mann-Whitney U test was used for further pairwise comparisons. The $\chi^{2}$ test was used for comparing the qualitative variables. A p-value $\leq 0.05$ was accepted as statistically significant.

\section{Results}

Table 1 presents the anthropometric details and $\mathrm{CBC}$ results of patients in the POAG, PACG, and control groups. The median age was $70(40-85)$ years in the POAG group $(\mathrm{n}=200), 57(42-89)$ years in the PACG group $(n=22)$, and $67(41-86)$ years in the control group $(\mathrm{n}=100)(\mathrm{p}=0.001)$. There were $49(49 \%)$ men in the control group, 92 men (46\%) in the POAG group, and 7 men $(32 \%)$ in the PACG group $(\mathrm{p}=0.342)$. The PACG group was younger than the POAG and control groups $(\mathrm{p}<0.05)$.

The difference in WBC, neutrophils, lymphocytes and NLR between POAG, PACG and control groups was not statistically significant $(\mathrm{p}=0.237, \mathrm{p}=0.249, \mathrm{p}=0.089$ and $\mathrm{p}=0.076$, respectively). A significant difference was found in terms of PLT and PLR levels between three groups ( $p<0.001$ and $p<0.001$, respectively).

We also compared the results between pairs of groups as follows: POAG and controls; PACG and controls; POAG and PACG. The values of platelets, NLR and PLR were significantly higher in the POAG group than in the control group $(\mathrm{p}=0.025$; $p=0.035$ and $p<0.001$, respectively). PLR and platelets were significantly higher in the PACG group than in the control group $(\mathrm{p}=$ $0.023 ; p=0.041$, respectively). Moreover, there were no differences in NLR or PLR between POAG and PACG ( $\mathrm{p}=0.869$ and $\mathrm{p}=0.559$, respectively). The PACG group was significantly younger than the POAG group $(\mathrm{p}=0.002)$. 
Tab. 2. Comparison of NLR and PLR between POAG and control.

\begin{tabular}{lccc}
\hline Variables & POAG $(\mathrm{n}=200)$ & Control $(\mathrm{n}=100)$ & $\mathrm{p}$ \\
\hline NLR & $2.19(0.79-13.23)$ & $2.08(0.20-6.73)$ & $0.035^{*}$ \\
PLR & $147.77(61.63-412.9)$ & $109.95(12.30-304.8)$ & $0.001^{*}$ \\
\hline \multicolumn{4}{l}{ POAG-primary open angle glaucoma; NLR - neutrophil-to-lymphocyte ratio; PLR } \\
- platelet-to-lymphocyte ratio; * $\mathrm{p}<0.05$
\end{tabular}

Tab. 3. Comparison of NLR and PLR between PACG and control.

\begin{tabular}{lrcc}
\hline Variables & \multicolumn{1}{c}{ PACG $(\mathrm{n}=22)$} & Control $(\mathrm{n}=100)$ & $\mathrm{p}$ \\
\hline NLR & $2.27(1.39-3.88)$ & $2.08(0.20-6.73)$ & 0.100 \\
PLR & $139.63(82-269.2)$ & $109.95(12.30-304.8)$ & $0.023^{*}$ \\
\hline
\end{tabular}

PACG - primary angle closure glaucoma; NLR - neutrophil-to-lymphocyte ratio; PLR - platelet-to-lymphocyte ratio; $* \mathrm{p}<0.05$

Tab. 4. Comparison of NLR and PLR between POAG and PACG.

\begin{tabular}{lcrc}
\hline Variables & \multicolumn{1}{c}{ POAG $(\mathrm{n}=200)$} & \multicolumn{1}{c}{ PACG $(\mathrm{n}=22)$} & $\mathrm{p}$ \\
\hline NLR & $2.19(0.79-13.23)$ & $2.27(1.39-3.88)$ & 0.869 \\
PLR & $147.77(61.63-412.9)$ & $139.63(82-269.2)$ & 0.559 \\
\hline
\end{tabular}

POAG - primary open angle glaucoma; PACG - primary angle closure glaucoma; NLR - neutrophil-to-lymphocyte ratio; PLR - platelet-to-lymphocyte ratio

\section{Discussion}

We found that platelets and PLR levels were significantly higher in both the POAG and PACG groups than in the control group, whereas NLR levels were significantly higher only when comparing the POAG and control groups. NLR levels were also higher in the PACG group when compared to the control group; however, this difference was not statistically significant.

Neutrophils, lymphocytes and platelets are easily determined by a CBC assay. These blood cells serve a significant role in the control of inflammation. The systemic inflammation is associated with alterations in the quantity and composition of circulating blood cells such as thrombocytosis, lymphopenia and neutrophilia (29). Since NLR and PLR are calculated manually from neutrophils, lymphocytes and platelet levels, they are supposed to be worthwhile in the management of diseases related to inflammation.

NLR and PLR values were not widely being used in ocular diseases until their positive role in determining the severity of certain diseases was demonstrated. For instance, Karaca et al demonstrated that the NLR value was capable of predicting keratoconus progression in patients (30). Furthermore, Huang et al evaluated serum cytokine levels in patients with POAG and claimed that higher cytokines were associated with optic neuropathy in POAG (31).

However, relatively few studies have evaluated NLR and PLR in patients with glaucoma. Similar to our results, Ozgonul et al observed higher levels of NLR and PLR in the POAG group than in the control group (25). Moreover, Li et al demonstrated that NLR values were higher in patients with PACG than in controls (32). In agreement with Li's findings, we also observed higher NLR values in the PACG group when compared to the control group; however, our findings did not reach the significance level.

Platelets are blood cells that initiate the release of inflammatory mediators by interacting with endothelial cells and leukocytes. In the process of inflammation, megakaryocytic proliferation and relative thrombocytosis occur. Moreover, increased platelet counts may be more likely to form platelet-rich vascular thrombi on atherosclerotic plaques in the coronary artery and/or peripheral arterial tree (33). PLR has been found to be an independent indicator of adverse medical conditions such as tissue ischemia in patients with acute myocardial infarction (34). One of the possible molecular mechanisms for POAG is thought to be ischemia in the optic nerve. Furthermore, chronic circulatory impairment in the optic nerve head $(\mathrm{ONH})$ causes irreversible damage to the retinal ganglion cells $(6,35)$. In our study, according to the finding of higher platelets and PLR values in the POAG and PACG groups, we suggest that platelets may play a role in both initiating inflammation and micro-plaque formation, thereby altering the blood supply of ONH.

The main limitation of this study is the lack of data on patients' history of medicine intake, co-morbidities, inflammation markers and/or inflammatory cytokines (e.g., CRP, MCP-1). The study design is another limitation since it is a retrospective and single-centre study. Moreover, the lack of measurements from Doppler ultrasonography and fundus fluorescein angiographic findings evaluating the presence of any abnormalities in vascular structures associated with the optic nerve represents another limitation.

In conclusion, higher serum platelets and PLR levels indicate that systemic inflammatory responses may be involved in both POAG and PACG pathogenesis. The use of systemic anti-inflammatory as well as anti-coagulant agents in glaucoma patients should be further investigated while managing glaucomatous optic neuropathies.

\section{Learning points}

- This is the first clinical study evaluating the neutrophil-tolymphocyte ratio (NLR) and platelet-to-lymphocyte ratio (PLR) in both the primary open-angle glaucoma (POAG) and primary angle-closure glaucoma (PACG) in a single study.

- The neutrophil-to-lymphocyte ratio (NLR) and platelet-tolymphocyte ratio (PLR) are inexpensive biomarkers obtained from complete blood count.

- It is found that PLT and PLR levels were significantly higher in both the POAG and PACG groups than in the control group,

- Vascular dysfunction that causes ischemia in the optic nerve is one of the major causes for the development and progression of glaucoma. Similarly, the higher levels of platelets and PLR may also indicate that systemic inflammation may play a role in the pathogenesis of POAG and PACG.

- This study adds new insight to the possible mechanisms of glaucoma.

- Future studies should be planned for the affectivity of antiinflammatory as well as anti-coagulant treatment strategies in glaucoma patients

\section{References}

1. Weinreb RN, Khaw PT. Primary open-angle glaucoma. Lancet (London, England) 2004; 363 (9422): 1711-1720. DOI: 10.1016/S0140-6736 (04)16257-0. 
2. Pan Y, Varma R. Natural history of glaucoma. Indian J Ophthalmol 2011; 59 Suppl: S19-23. DOI: 10.4103/0301-4738.73682.

3. Weinreb RN, Aung T, Medeiros FA. The pathophysiology and treatment of glaucoma: a review. JAMA 2014; 311 (18): 1901-1911. DOI: 10.1001/ jama.2014.3192.

4. Distelhorst JS, Hughes GM. Open-angle glaucoma. Am Fam Physician 2003; 67 (9): 1937-1944.

5. Sun X, Dai Y, Chen Y et al. Primary angle closure glaucoma: What we know and what we don't know. Prog Retin Eye Res 2017; 57: 26-45. DOI: 10.1016/j.preteyeres.2016.12.003.

6. Hayreh SS. Factors influencing blood flow in the optic nerve head. J Glaucoma. 1997; 6 (6): 412-425.

7. Wey S, Amanullah S, Spaeth GL, Ustaoglu M, Rahmatnejad K, Katz LJ. Is primary open-angle glaucoma an ocular manifestation of systemic disease? Graefes Arch Clin Exp Ophthalmol 2019; 257 (4): 665-673. DOI: 10.1007/s00417-019-04239-9.

8. Maruyama I, Ohguro H, Ikeda Y. Retinal ganglion cells recognized by serum autoantibody against gamma-enolase found in glaucoma patients. Invest Ophthalmol Vis Sci 2000; 41 (7): 1657-1665.

9. Yang J, Tezel G, Patil R V, Romano C, Wax MB. Serum autoantibody against glutathione $\mathrm{S}$-transferase in patients with glaucoma. Invest Ophthalmol Vis Sci 2001; 42 (6): 1273-1276.

10. Tezel G, Edward DP, Wax MB. Serum autoantibodies to optic nerve head glycosaminoglycans in patients with glaucoma. Arch Ophthalmol (Chicago, Ill 1960). 1999; 117 (7): 917-924. DOI: 10.1001/archopht.117.7.917.

11. Mousa A, Kondkar AA, Al-Obeidan SA et al. Association of total antioxidants level with glaucoma type and severity. Saudi Med J 2015; 36 (6): 671-677. DOI: 10.15537/smj.2015.6.10697.

12. Atalay K, Kaldirim Erdogan H, Kirgiz A, Asik Nacaroglu S. Predictive role of neutrophil-to-lymphocyte ratio and platelet-to-lymphocyte ratio in normal-tension glaucoma. Med Hypotheses 2017; 103: 54-56. DOI: 10.1016/j.mehy.2017.04.001

13. Hammad M, Shehata OZ, Abdel-Latif SM, El-Din AMM. Neutrophil/lymphocyte ratio and platelet/lymphocyte ratio in Behcet's disease: which and when to use? Clin Rheumatol 2018; 37 (10): 2811-2817. DOI: 10.1007/s10067-018-4194-z.

14. Gasparyan AY, Ayvazyan L, Mukanova U, Yessirkepov M, Kitas GD. The Platelet-to-Lymphocyte Ratio as an Inflammatory Marker in Rheumatic Diseases. Ann Lab Med 2019; 39 (4): 345-357. DOI: 10.3343/ alm.2019.39.4.345.

15. Wang Q, Ma J, Jiang Z, Ming L. Prognostic value of neutrophil-tolymphocyte ratio and platelet-to-lymphocyte ratio in acute pulmonary embolism: a systematic review and meta-analysis. Int Angiol 2018; 37 (1): 4-11. DOI: $10.23736 / \mathrm{S} 0392-9590.17 .03848-2$.

16. Chung JH, Lim J, Jeong JH, Kim KR, Park CW, Lee SH. The significance of neutrophil to lymphocyte ratio and platelet to lymphocyte ratio in vestibular neuritis. Laryngoscope 2015; 125 (7): E257-61. DOI: 10.1002/ lary.25204.

17. Shi G, Zhao J-W, Ming L. Clinical significance of peripheral blood neutrophil-lymphocyte ratio and platelet- lymphocyte ratio in patients with asthma. Nan Fang Yi Ke Da Xue Xue Bao 2017; 37 (1): 84-88.

18. Fan Z, Hao L, Chuanyuan T et al. Neutrophil and Platelet to Lymphocyte Ratios in Associating with Blood Glucose Admission Predict the Functional Outcomes of Patients with Primary Brainstem Hemorrhage. World Neurosurg 2018; 116: e100-e107. DOI: 10.1016/j.wneu.2018.04.089.

19. Dursun A, Ozturk S, Yucel $\mathbf{H}$ et al. Association of neutrophil/lymphocyte ratio and retinal vein occlusion. Eur J Ophthalmol 2015; 25 (4): 343-346. DOI: 10.5301/ejo.5000570.
20. Guclu H, Ozal SA, Pelitli Gurlu V, Birgul R. Elevated Neutrophil Lymphocyte Ratio in Recurrent Optic Neuritis. J Ophthalmol 2015; 2015: 758687. DOI: $10.1155 / 2015 / 758687$.

21. Ozgonul C, Sertoglu E, Ayyildiz $O$ et al. Novel biomarkers for patients with idiopathic acute anterior uveitis: neutrophil to lymphocyte ratio and platelet to lymphocyte ratio. Int J Ophthalmol 2017; 10 (2): 262-266. DOI: 10.18240/ijo.2017.02.13.

22. Inanc M, Tekin K, Budakoglu O, Ilhan B, Aydemir O, Yilmazbas P. Could Platelet Indices and Neutrophil to Lymphocyte Ratio Be New Biomarkers for Differentiation of Arteritic Anterior Ischemic Neuropathy from Non-Arteritic Type? Neuroophthalmology 2018; 42 (5): 287-294. DOI: 10.1080/01658107.2017.1405995.

23. Ozgonul C, Sertoglu E, Mumcuoglu T, Ozge G, Gokce G. Prediction of Pseudoexfoliation Syndrome and Pseudoexfoliation Glaucoma by Using Neutrophil to Lymphocyte Ratio and Platelet to Lymphocyte Ratio. Ocul Immunol Inflamm 2016; 24 (6): 665-670. DOI: 10.3109/09273948.2015.1063671.

24. Petropoulos IN, Kamran S, Li Y et al. Corneal Confocal Microscopy: An Imaging Endpoint for Axonal Degeneration in Multiple Sclerosis. Invest Ophthalmol Vis Sci 2017; 58 (9): 3677-3681. DOI: 10.1167/iovs.17-22050.

25. Ozgonul C, Sertoglu E, Mumcuoglu T, Kucukevcilioglu M. Neutrophil-to-Lymphocyte Ratio and Platelet-to-Lymphocyte Ratio as Novel Biomarkers of Primary Open-Angle Glaucoma. J Glaucoma 2016; 25 (10): e815-e820. DOI: 10.1097/IJG.0000000000000392.

26. Foster PJ, Buhrmann R, Quigley HA, Johnson GJ. The definition and classification of glaucoma in prevalence surveys. Br J Ophthalmol 2002; 86 (2): 238-242. DOI: 10.1136/bjo.86.2.238.

27. Chakrabarty L, Joshi GD, Chakravarty A, Raman G V, Krishnadas SR, Sivaswamy J. Automated Detection of Glaucoma From Topographic Features of the Optic Nerve Head in Color Fundus Photographs. J Glaucoma 2016; 25 (7): 590-597. DOI: 10.1097/IJG.0000000000000354.

28. Rogers TW, Jaccard N, Carbonaro F et al. Evaluation of an AI system for the automated detection of glaucoma from stereoscopic optic disc photographs: the European Optic Disc Assessment Study. Eye (Lond). July 2019. DOI: $10.1038 / \mathrm{s} 41433-019-0510-3$.

29. Gabay C, Kushner I. Acute-phase proteins and other systemic responses to inflammation. N Engl J Med. 1999; 340 (6): 448-454. DOI: 10.1056/ NEJM199902113400607.

30. Karaca EE, Ozmen MC, Ekici F, Yuksel E, Turkoglu Z. Neutrophilto-lymphocyte ratio may predict progression in patients with keratoconus. Cornea 2014; 33 (11): 1168-1173. DOI: 10.1097/ICO.0000000000000260.

31. Huang P, Qi Y, Xu Y-S et al. Serum cytokine alteration is associated with optic neuropathy in human primary open angle glaucoma. J Glaucoma 2010; 19 (5): 324-330. DOI: 10.1097/IJG.0b013e3181b4cac7.

32. Li S, Cao W, Han J, Tang B, Sun X. The diagnostic value of white blood cell, neutrophil, neutrophil-to-lymphocyte ratio, and lymphocyte-tomonocyte ratio in patients with primary angle closure glaucoma. Oncotarget 2017; 8 (40): 68984-68995. DOI: 10.18632/oncotarget.16571.

33. Stoll G, Nieswandt B. Thrombo-inflammation in acute ischaemic stroke - implications for treatment. Nat Rev Neurol. July 2019. DOI: 10.1038/ s41582-019-0221-1.

34. Ozcan Cetin EH, Cetin MS, Aras D et al. Platelet to Lymphocyte Ratio as a Prognostic Marker of In-Hospital and Long-Term Major Adverse Cardiovascular Events in ST-Segment Elevation Myocardial Infarction. Angiology 2016; 67 (4): 336-345. DOI: 10.1177/0003319715591751.

35. Flammer J. The vascular concept of glaucoma. Surv Ophthalmol 1994; 38 Suppl: S3-6.

Received June 19, 2020. Accepted October 26, 2020. 